\title{
WAVELET DECOMPOSITION APPROACH FOR UNDERSTANDING TIME-VARYING RELATIONSHIP OF FINANCIAL SECTOR VARIABLES: A STUDY OF THE INDIAN STOCK MARKET
}

DOI: $10.22367 / \mathrm{mcdm} / 2020.15 .03$

Received: 17.11.2020 | Revised: 26.04.2021 | Accepted: 14.09.2021.

\begin{abstract}
In this paper, we study the effect of overall stock market sentiment in India on sectoral indices and on individual stock prices in terms of co-movement, dependence and volatility transmission along with the magnitude and persistence of the effects. The study uses wavelet decomposition framework for breaking down different financial time series into time-varying components. Quantile Regression, Wavelet Multiple Correlation and Cross-Correlation analysis, and Diebold-Yilmaz spillover analysis are then applied to investigate the nature of dependence, association, and spillover dynamics. For further focus, we have considered different time periods separately to identify the effect of market phases. Interesting results are obtained with respect to persistence of shocks, both across and within time periods. These have implications with respect to understanding market behavior and also perception of sectors and stocks.
\end{abstract}

Keywords: Wavelet Decomposition, SENSEX, Quantile Regression, Wavelet Multiple Correlation, Wavelet Multiple Cross Correlation, Diebold-Yilmaz Spillover.

\footnotetext{
IT \& Analytics Area, Institute of Management Technology Hyderabad, Shamshabad, Hyderabad-501218, Telangana, India, e-mail: indranil@imthyderabad.edu.in, ORCID: 00000001-7064-4774.

** Centre for Knowledge, Ideas and Development Studies, KnIDS, Kolkata, India, e-mail: tamal5302@yahoo.com, ORCID: 0000-0002-5086-6019.
} 


\section{Introduction}

Examination of behavioral patterns, causal nexus and volatility contagion in global stock markets has gained considerable attention among researchers. Proper understanding of such relationships is crucial as they have implications in investments, portfolio formation, financial flows and policy making (Glensk, Ganczarek-Gamrot and Trzpiot, 2013; Juszczuk, Kaliszewski and Miroforidis, 2017; Ghosh and Datta Chaudhuri, 2018; Ghosh and Datta Chaudhuri, 2019). The global financial crisis of 2008-09 showed that in today's globalized world, disruptions in one part of the globe can easily spread to other parts. It is thus important to study the inherent characteristics of market sentiment and its influence on other assets for effective risk management. The present study attempts to analyze the effect of overall stock market sentiment in India on sectoral indices and also on individual stock price movements in terms of co-movement, dependence and volatility transmission along with the magnitude and persistence of the effects. The analysis is made for four distinct time periods in the Indian stock market, namely: pre-crisis (2007-08), crisis (2008-09), bearish period (2015) and a bullish phase (2016). SENSEX returns have been chosen to represent overall market sentiment. Sectoral returns are represented by returns from the MID CAP Index and SMALL CAP Index. Further, daily stock returns of six different companies, namely: Bharat Forge Limited (BF), Cipla Limited (CP), Godrej Consumer Products Limited (GD), Biocon Limited (BC), Tata Consultancy Services Limited (TCS) and Glenmark Pharmaceutical Limited (GM) belonging to separate industrial sectors are analyzed to evaluate their co-movements with market sentiment and also the spillover dynamics during the four periods. The analysis can easily be extended to understand stock price movements of other companies.

Beta of Capital Asset Pricing Model (CAPM) has garnered a lot of interest in the literature. While some have looked at the time-varying properties of Beta, others have focused on "Good Beta, Bad Beta". No paper on stock price prediction has been able to ignore Beta in their model specification and analysis. Stocks have been characterized by Beta, and portfolio formation and realignment has been based on its magnitude. Although myriads of research work have attempted to model the interplay stock returns with market returns, the persistence of shocks cannot be explained by the CAPM model.

Modeling volatility has received strong traction in the literature which has resulted in ARCH, GARCH, DCC-GARCH, etc. framework-driven work to analyze the same (Datta Chaudhuri and Ghosh, 2015, 2016; Ghosh, Sanyal and Jana, 2021). As Beta is covariance divided by variance, researchers have applied 
this class of models, as there is volatility in the time series of returns. The purpose of this paper is to measure not the extent of market-wide shocks on individual stock returns or sectoral returns, but the persistence of the shocks. For this, we go granular and try to identify associations between market returns, individual stock returns, and sectoral returns in the short run, medium term and long term. Measurements of the extent of spillover of shocks and the duration of the shocks are the focus of the paper. We are not focusing on whether stocks are aggressive, neutral, or defensive; we are interested to know whether the responsiveness of their returns to market shocks is temporary or prolonged. In terms of length of persistence of shocks, our quest is also to understand whether market-wide shocks affect different sectors differently. If so, then this helps in portfolio formation.

The major contributions of the present study can be segregated into three broad strands. It first attempts to contribute to the literature by analyzing the dynamic interplay in terms of inspecting the duration of the same at the short, medium, and long run duration at granular level. The interplay has been decoded through careful investigation of dependence structure, co-movement dynamics, and volatility contagion effects. The methodological frameworks resort to quantile regression, wavelet driven correlation, and Diebold-Yilmaz spillover analysis in conjunction with wavelet decomposition to capture dependence, co-movement, and spillover relationship, respectively. Second, the research aims to expound the interplay in four specific regimes linked explicitly to the global financial crisis. Unlike the majority of previous work dedicated to examining interrelationships in the pre-crisis, crisis, and post-crisis phases at aggregate level, the present study further bifurcates the post-crisis phase into downswing and upswing phases for drawing inferences. The regime-driven exploration in association with wavelet-based time-frequency modeling delivers key insights and rationalizes the positioning of the paper with respect to past literature. The third contribution of the study lies in the selection of financial variables in Indian context. Again, dominance of large sector stocks while analyzing market sentiment, transmission of volatility, shocks is apparent in the literature. However, there is a dearth of research in assessment of mid- and small-cap sectors. The underlying work systematically fills the gap through incorporating both these sectors, alongside company-specific stocks, in the research agenda.

Performance of sectors of an economy reflects the demand pattern of the consumers, both national and global. This manifests itself in the performance of the companies in the different sectors, and gets reflected in their share prices. The business scenario is dynamic; companies of various sizes are trying to survive and grow. Companies of various sizes can be classified by their market 
capitalization and we have large cap, mid cap and small cap companies. Our focus in this paper is also on whether overall market-wide shocks affect companies of various sizes differently. Companies whose share prices take a beating, may find it hard in the short run to raise resources from the market, which in turn can adversely affect their growth prospects. Thus it is important to measure the extent of spillover of the shocks and also the duration of the shocks across sectors and companies.

The majority of financial time series during the said time periods have been observed to exhibit high degree of nonlinear and nonparametric behavior. Nonparametric behavior of the said series is a reflection of the violation of the normal distribution. Standard econometric and statistical approaches often fail to derive any significant insights from such data. Discrete Wavelet Transformation (DWT) is a nonparametric tool which is capable of modelling any high volatile and nonlinear time series in combined time and scale domain. It decomposes the original time series into a set of linear and nonlinear granular components accounting for different time horizons in an effective way. We use Maximal Overlap Discrete Wavelet Transformation (MODWT) algorithm, which is a variant of the DWT technique, and possesses several advantages over the latter in the decomposition process.

In this paper, we decompose each time series, and go granular for better understanding of the relationships within time phases. The following methods are used, on the decomposed series in different contexts, to analyze the time-varying pattern of the relationships in the Indian stock market.

1. Quantile Regression is applied in conjunction with wavelet decomposition to understand the dependence of MID CAP index returns and SMALL CAP index returns on market sentiment measured in terms of SENSEX returns.

2. Time-varying nature of association between SENSEX returns and individual company stock returns are analyzed through a separate framework called Wavelet Multiple Correlation (WMC) and Wavelet Multiple Cross Correlation (WMCC).

3. Volatility spillover of market sentiment on sectoral returns and company stock returns are explored through the Diebold-Yilmaz volatility spillover index on decomposed time components to understand time-varying characteristics of the same.

It may be noted that there exists a body of literature that explores the dynamics of co-integration and interrelationship of financial markets during the pre-crisis and crisis periods. However, the majority of these studies are either based on data of developed economies or confined to frameworks similar to CAPM. We have not come across granular wavelet-based research models to 
decode time-varying behavioral patterns of stock markets of developing economies during such time periods. The present paper attempts to fill this gap.

The rest of the paper is organized as follows. Section 2 is a discussion of the literature related to our research objectives and models. Section 3 presents the data profile and key statistical properties of the same. The research methodology is explained in Section 4, and Section 5 discusses the overall findings. Section 6 concludes the paper.

\section{Literature review}

As the objective of this paper is to examine the time-varying interaction among the financial sector variables, this section briefly outlines some recent work in financial modeling using different methodological frameworks. A careful and thorough review of cognate literature assists in identifying the trend and prevailing gaps. It should be noted that the effects of global financial crisis on markets have been studied by Gao and Mei (2019); Nikkinen, Piljak and Rothovius (2020); Bessler et al. (2021); Yamani (2021). Nevertheless, these studies mostly relied upon orthodox statistical and econometric modeling which limit the scope of finding a dynamic evolutionary pattern. To overcome the challenge, the present study resorts to wavelet-driven modeling. We have restricted the review to wavelet-driven frameworks for modeling temporal relationship specifically, as the primary objective of the present paper is to explicate the dynamics in varying timescales.

Ghosh and Datta Chaudhuri (2016) applied wavelet decomposition framework for studying interplay among historic and implied volatility indices of Indian and US markets. The results revealed time-varying nature of bidirectional and unidirectional causal structure which were effectively utilized for forecasting individual financial time series using machine learning models.

Jammazi et al. (2017) explored time-varying nature of causality structure between oil price change and stock returns of six oil-importing countries using wavelet analysis and dynamic causality test for assessing dependence in multiresolution framework. The overall outcome suggested the existence of strong causal links in the short run during the global financial crisis.

Liu et al. (2017) studied the volatility spillover phenomenon between oil and stock markets employing time-varying granular model comprising wavelet decomposition and GARCH-BEKK tool. Findings indicated that spillovers between oil and S\&P 500 index reduced in long run, while a completely opposite behavior was prevalent between the MICEX index and oil market during the entire time span. 
Das et al. (2018) assessed the nexus of Asian gold spot markets of China, India, Indonesia, South Korea, Thailand and Vietnam through Wavelet Coherence (WC), Wavelet Multiple Correlation (WMC) and Wavelet Multiple Cross Correlation (WMCC) models. Strong positive co-movement structure was found and Thailand emerged as the potential market leader.

Das et al. (2018) applied Wavelet Local Correlation (WLC) to examine the nature of contagion, interrelationship, and changes in association among emerging stock markets after the global financial crisis. The findings implied the presence of weaker contagion among Latin American emerging markets, significant contagion between European and Middle East markets, and insignificant long-run association between them post crisis.

Das and Kannadhasan (2018) explored the dependence of Bitcoin price on different macroeconomic factors, stock index, economic policy uncertainty, implied volatility, gold and crude oil price, using wavelet-based multi-scale approach. Findings revealed nonexistence of dependence of Bitcoin price on those factors in short run; however, significant dependence was present in the medium to long run.

Polanco-Martiez et al. (2018) carried out an exploratory research to determine the interplay between EU peripheral stock indices and the S\&P Europe 350 index during pre-crisis and crisis periods through wavelet-based rolling correlation and nonlinear Granger causality test. The findings suggested the existence of stronger association and higher causal interaction in the crisis period than in the pre-crisis period.

Ghosh and Datta Chaudhuri (2019) examined pairwise association and causality between four different pairs of financial assets, namely: CBOEVIX (Implied volatility of US market) and INDIAVIX (Implied volatility of Indian market), Rupee-Dollar exchange rate and Crude oil return, DJIA return and IT sectoral return of India, and SENSEX and METAL sector return of India though MODWT-based granular Dynamic Conditional Correlation (DCC) - GARCH, Asymmetric Dynamic Conditional Correlation (ADCC) - GARCH models and the Diks-Panchenko test. The results indicated the existence of significant association at different timespan and asymmetric causal interaction. They also investigated volatility contagion dynamics of eight assets together using MODWT-based Diebold-Yilmaz spillover analysis and found the dominance of CBOEVIX in the long run.

Mishra et al. (2019) used wavelet-driven quantile regression framework to decode the interrelationship between the global crude oil price fluctuations and the Dow Jones Islamic stock index. Their findings indicated that short oil price fluctuation had positive influence on stock index, while the impact turned out to be adverse in the long run. 
Mo et al. (2019) explored the influence of crude oil prices on the economic growth of the BRICS countries using wavelet-driven quantile-on-quantile tests. Positive effects on economic growth emerged, though heterogeneity across countries, periods, and quantiles were observed.

Dai et al. (2020) examined the dynamic dependence and risk contagion among oil, gold, and US foreign exchange markets in multiscale manner through deploying wavelet and time-varying vine-copula and vine-copula quantile regression approaches. Their results implied that the US foreign exchange market possessed stronger influence in the short run, while gold markets, in the medium run. Asymmetry in the risk contagion effect was also observed.

Ghosh, Sanyal and Jana (2021) resorted to continuous wavelet transformation-driven wavelet coherence analysis to model the dynamic dependence structure of financial and energy markets. The presence of long and medium run co-movement among the assets was discovered. They further combined MODWT and DCC-GARCH in an integrated framework to successfully estimate hedge ratios across the time scales.

Goodell and Goutte (2020) explored the co-movement of global COVID-19 mortality and Bitcoin price using wavelet coherence. The results indicated a strong negative association prevailing between the underlying variables.

Jiang and Yoon (2020) used wavelet multiscale decomposition and wavelet coherence analysis to inspect interaction of oil and stock markets of oil-importing and oil-exporting nations. The outcome revealed that stock markets of oil-exporting nations were relatively more affected by oil markets than those of the oil-importing nations.

Sharif, Aloui and Yarovaya (2020) used wavelet coherence and combined framework of discrete wavelet transformation and Granger causality to evaluate the dynamic interplay among daily COVID-19 infections in the US, US stock market (DJIA), geopolitical risk (GPR) in the US, economic political uncertainty (EPU) in the US, and WTI crude oil price. The overall findings suggested the presence of asymmetric association and a stronger penetration of the impact of COVID-19 infections on GPR and EPU of the US.

Thus it is amply apparent that wavelet-driven approaches have been extremely effective in modeling different financial time series data and in mining association among heterogeneous assets in time-varying manner. There is clear trend using these tools in discovering critical interaction structure at challenging times for practical implications including strategic management and policy formulations. These methodologies can delve quite effectively into financial markets with a high degree of volatility and nonlinearity at granular level. It should be noted that wavelet-driven frameworks have emerged to be 
extremely successful in predictive modeling of financial markets as well (Ghosh and Datta Chaudhuri, 2017; Ghosh, Sanyal and Jana, 2018; Ghosh and Datta Chauduri, 2019; Ghosh, Jana and Sanyal, 2019). Therefore resorting to wavelet-driven frameworks to accomplish the current research endeavors is truly justified. On the other hand, in terms of gap analysis and positioning the underlying work, the aforesaid review provides sufficient information and scope. It is amply evident that despite the prevalence of a substantial amount of research dedicated to wavelet-driven dynamic evaluation of interaction patterns, the scantiness of research concentrated on the Indian context specifically is apparent. Empirical studies to categorically evaluate the nature of association at the outset of black swan events such as the global financial crisis have largely been made on aggregate series which fall short of decoding short, medium, and long run patterns distinctly. Moreover, post-crisis assessment has not penetrated to a deeper level, to the best of our knowledge. The current paper effectively addresses the existing gaps through a proper selection of methodological frameworks, variables and regimes. Recently, the examination of the behavioral difference in reactions of stock markets to the global financial crisis and the COVID-19 pandemic has steadily garnered attention (Chang, McAleer and Wang, 2020; Choi, 2021). Nonetheless, the said analysis is beyond the scope of the current research.

\section{Data profile}

In order to analyze the dynamics of the Indian stock market, two different set of modeling exercises have been conducted during the pre-crisis, crisis and post-crisis periods.

In the first experiment, we have considered daily returns of SENSEX, BSE MID CAP INDEX (MID CAP) and BSE SMALL CAP INDEX (SMALL CAP) for understanding of the nature of behavior and nexus of sectoral indices in the Indian stock market. The study attempts to capture the influence of market sentiment on returns of MID CAP and SMALL CAP indices, assess the volatility dynamics, and measure the extent of spillovers.

The other experiment analyzes the co-movement of SENSEX return with selected Indian company stock returns, namely: Bharat Forge (BF), Cipla (CP), Godrej Consumer Products (GD), Biocon (BC), Tata Consultancy Service (TCS) and Glenmark (GM).

Daily observations on the variables from January 2007 to January 2008 have been considered for pre-crisis analysis; crisis period analysis has been performed on daily observations from February 2008 to March 2009; and daily data for two 
separate time periods, March 2015 to February 2016 (representing downswing) and March 2016 to November 2016 (representing upswing) have been considered.

We have estimated the key statistical properties of our dataset across different phases using well-known statistical tests. Tables 1-8 summarize the results.

Table 1: Test of normality and stationarity of SENSEX and sectors during Pre-Crisis

\begin{tabular}{|l|r|r|r|r|}
\hline \multicolumn{1}{|c|}{ Series } & Jarque-Bera Test & Shapiro-Wilk Test & \multicolumn{1}{c|}{ Terasvirta Test } & \multicolumn{1}{c|}{ ADF Test } \\
\hline SENSEX & $41.32 * * *$ & $0.9705^{* * *}$ & $9.39 * * *$ & $-18.97 * *$ \\
\hline MID CAP & $36.69^{* * *}$ & $0.9138^{* * *}$ & $10.34 * * *$ & $-16.17 * * *$ \\
\hline SMALL CAP & $37.59 * * *$ & $0.9203 * * *$ & $10.08 * * *$ & $-16.98 * * *$ \\
\hline
\end{tabular}

$* * *$ Significant at $1 \%$ level of significance.

Table 2: Test of normality and stationarity of SENSEX and sectors during Crisis

\begin{tabular}{|l|r|r|r|r|}
\hline \multicolumn{1}{|c|}{ Series } & \multicolumn{1}{c|}{ Jarque-Bera Test } & Shapiro-Wilk Test & \multicolumn{1}{c|}{ Terasvirta Test } & \multicolumn{1}{c|}{ ADF Test } \\
\hline SENSEX & $48.76^{* * *}$ & $0.9640^{* * *}$ & $10.85^{* * * *}$ & $-17.58^{* * *}$ \\
\hline MID CAP & $45.72 * * *$ & $0.9189^{* * *}$ & $11.32^{* * * *}$ & $-15.84 * * *$ \\
\hline SMALL CAP & $51.33^{* * *}$ & $0.9239 * * *$ & $10.57 * * *$ & $-16.31 * * *$ \\
\hline
\end{tabular}

*** Significant at $1 \%$ level of significance.

Table 3: Test of normality and stationarity of SENSEX and sectors during Downswing (Post-Crisis)

\begin{tabular}{|l|r|r|r|r|}
\hline \multicolumn{1}{|c|}{ Series } & Jarque-Bera Test & Shapiro-Wilk Test & \multicolumn{1}{c|}{ Terasvirta Test } & \multicolumn{1}{c|}{ ADF Test } \\
\hline SENSEX & $39.15^{* * *}$ & $0.9146^{* * *}$ & $9.58^{* * *}$ & $-18.56^{* * *}$ \\
\hline MID CAP & $34.43^{* * *}$ & $0.8977 * * *$ & $10.67 * * *$ & $-16.73 * * *$ \\
\hline SMALL CAP & $36.82 * * *$ & $0.8860 * * *$ & $9.92 * * *$ & $-17.05 * * *$ \\
\hline
\end{tabular}

*** Significant at $1 \%$ level of significance.

Table 4: Test of normality and stationarity of SENSEX and sectors during Upswing (Post-Crisis)

\begin{tabular}{|l|r|r|r|r|}
\hline \multicolumn{1}{|c|}{ Series } & Jarque-Bera Test & Shapiro-Wilk Test & \multicolumn{1}{c|}{ Terasvirta Test } & \multicolumn{1}{c|}{ ADF Test } \\
\hline SENSEX & $49.07 * * *$ & $0.9258^{* * *}$ & $10.17 * * *$ & $-18.17 * *$ \\
\hline MID CAP & $47.24 * * *$ & $0.9406 * * *$ & $10.89 * * *$ & $-16.59 * * *$ \\
\hline SMALL CAP & $43.35 * * *$ & $0.9188^{* * * *}$ & $10.37 * * *$ & $-17.36^{* * * *}$ \\
\hline
\end{tabular}

*** Significant at $1 \%$ level of significance.

Significant Jarque-Bera and Shapiro-Wilk test statistics across all distinct time phases imply that none of the financial variables abide by normal distribution. Likewise significant Terasvirta test statistics signify a clear presence of nonlinear behavior in daily returns of three assets. Lastly, the Augmented Dickey Fuller (ADF) test indicates that all the series exhibit stationary behavior. 
Table 5: Test of normality and stationarity of SENSEX and six company stocks during Pre-Crisis

\begin{tabular}{|l|r|r|r|r|}
\hline \multicolumn{1}{|c|}{ Series } & Jarque-Bera Test & Shapiro-Wilk Test & \multicolumn{1}{c|}{ Terasvirta Test } & \multicolumn{1}{c|}{ ADF Test } \\
\hline SENSEX (SN) & $41.32 * * *$ & $0.9640 * * *$ & $9.39 * * *$ & $-18.97 * * *$ \\
\hline BHARAT FORGE (BF) & $44.58 * * *$ & $0.9709 * * *$ & $9.16 * * *$ & $-20.13 * * *$ \\
\hline CIPLA (CP) & $43.02 * * *$ & $0.9678 * * *$ & $9.28 * * *$ & $-19.46 * * *$ \\
\hline GODREJ (GD) & $51.16 * * *$ & $0.9711 * * *$ & $11.37 * * *$ & $-21.37 * * *$ \\
\hline BIOCON (BC) & $47.80 * * *$ & $0.9728 * * *$ & $10.61 * * *$ & $-18.84 * * *$ \\
\hline TCS (TC) & $52.40 * * *$ & $0.9719 * * *$ & $10.15 * * *$ & $-20.44 * * *$ \\
\hline GLENMARK (GM) & $40.39 * * *$ & $0.9621 * * *$ & $9.21 * * *$ & $-18.50 * * *$ \\
\hline
\end{tabular}

*** Significant at $1 \%$ level of significance.

Table 6: Test of normality and stationarity of SENSEX and six company stocks during Crisis

\begin{tabular}{|l|r|r|r|r|}
\hline \multicolumn{1}{|c|}{ Series } & Jarque-Bera Test & Shapiro-Wilk Test & Terasvirta Test & \multicolumn{1}{c|}{ ADF Test } \\
\hline SENSEX (SN) & $48.76 * * *$ & $0.9724 * * *$ & $10.85 * * *$ & $-17.58^{* * *}$ \\
\hline BHARAT FORGE (BF) & $49.34 * * *$ & $0.9733 * * *$ & $11.16 * * *$ & $-19.21 * * *$ \\
\hline CIPLA (CP) & $46.77 * * *$ & $0.9716^{* * *} *$ & $10.27 * * *$ & $-17.32 * * *$ \\
\hline GODREJ (GD) & $49.94 * * *$ & $0.9611 * * *$ & $10.06 * * *$ & $-18.17 * * *$ \\
\hline BIOCON (BC) & $50.13 * * *$ & $0.9745 * * *$ & $12.13 * * *$ & $-19.66^{* * *}$ \\
\hline TCS (TC) & $48.72 * * *$ & $0.9732 * * *$ & $11.48 * * *$ & $-17.43^{* * *}$ \\
\hline GLENMARK $(\mathrm{GM})$ & $45.60 * * *$ & $0.9603 * * *$ & $10.75 * * *$ & $-16.95 * * *$ \\
\hline
\end{tabular}

*** Significant at $1 \%$ level of significance.

Table 7: Test of normality and stationarity of SENSEX and six company stocks during Downswing (Post-Crisis)

\begin{tabular}{|l|r|r|r|r|}
\hline \multicolumn{1}{|c|}{ Series } & Jarque-Bera Test & Shapiro-Wilk Test & Terasvirta Test & \multicolumn{1}{c|}{ ADF Test } \\
\hline SENSEX (SN) & $39.15 * * *$ & $0.9718^{* * *}$ & $9.58 * * *$ & $-18.56^{* * *}$ \\
\hline BHARAT FORGE (BF) & $42.23 * * *$ & $0.9723 * * *$ & $9.92 * * *$ & $-20.31 * * *$ \\
\hline CIPLA (CP) & $41.79 * * *$ & $0.9706 * * *$ & $9.46 * * *$ & $-19.46 * * *$ \\
\hline GODREJ (GD) & $43.47 * * *$ & $0.9729 * * *$ & $10.07 * * *$ & $-21.54 * * *$ \\
\hline BIOCON (BC) & $42.28 * * *$ & $0.9648 * * *$ & $10.19 * * *$ & $-20.83 * * *$ \\
\hline TCS (TC) & $44.34 * * *$ & $0.9701 * *$ & $11.10 * * *$ & $-22.42 * * *$ \\
\hline GLENMARK (GM) & $38.75 * * *$ & $0.9613 * * *$ & $9.32 * * *$ & $-18.11 * * *$ \\
\hline
\end{tabular}

*** Significant at $1 \%$ level of significance.

Table 8: Test of normality and stationarity of SENSEX and six company stocks during Upswing (Post-Crisis)

\begin{tabular}{|l|r|r|r|r|}
\hline \multicolumn{1}{|c|}{ Series } & Jarque-Bera Test & Shapiro-Wilk Test & \multicolumn{1}{c|}{ Terasvirta Test } & \multicolumn{1}{c|}{ ADF Test } \\
\hline SENSEX (SN) & $49.07 * * *$ & $0.9713 * * *$ & $10.17 * * *$ & $-18.17 * * *$ \\
\hline BHARAT FORGE (BF) & $51.16 * * *$ & $0.9706 * * *$ & $11.58 * * *$ & $-19.10^{* * *}$ \\
\hline CIPLA (CP) & $47.82 * * *$ & $0.9643 * * *$ & $9.75 * * *$ & $-17.88^{* * *}$ \\
\hline GODREJ (GD) & $53.49 * * *$ & $0.9739 * * *$ & $12.29 * * *$ & $-20.38 * * *$ \\
\hline BIOCON (BC) & $48.18 * * *$ & $0.9685 * * *$ & $10.53 * * *$ & $-18.85 * * *$ \\
\hline TCS (TC) & $46.74 * * *$ & $0.9661 * * *$ & $10.01 * * *$ & $-17.76 * * *$ \\
\hline GLENMARK (GM) & $45.11 * * *$ & $0.9624 * * *$ & $9.89 * * *$ & $-17.68 * * *$ \\
\hline
\end{tabular}

*** Significant at $1 \%$ level of significance. 
Similar to findings of market sentiment and sectoral indices, daily returns of all six company stocks are found to be nonparametric (as the underlying series barely exhibits any sign of normal distribution), nonlinear and stationary in nature across all time phases. As the key endeavor of this research is to identify the time-varying characteristics, i.e. persistence, dependence, and volatility, wavelet-based time series decomposition approach has been used. Wavelet decomposition framework can simultaneously carry out inspection of behavioral characteristics of financial data in both different time and scales. It can also effectively model time series exhibiting high degree of non-parametric, nonlinear, and nonstationary behavior with great accuracy. Hence the deployment of wavelet-based examination frameworks in this paper is well justified as it serves the research purpose and is appropriate for dealing with financial data under consideration.

\section{Methodology}

This section outlines the detailed operational processes of the methodological frameworks used sequentially. As stated, we have deployed quantile regression, wavelet correlation, and Diebold-Yilmaz spillover analysis in conjunction with wavelet decomposition to accomplish research goals. The rationale behind the use of different research tools lies in the process of analyzing the dependence structure, co-movement, and volatility spillover separately. Unlike the plethora of previous research, we have not restricted the investigation to one particular form of interrelationship.

Wavelet Decomposition: Through wavelet transformation, the original series is expressed as a set of superimposed wavelets or orthogonal components. It generates father $(\varphi(t))$ and mother $(\psi(t))$ wavelets in scale-wise manner by translating and dilating the original function $(f(t))$. The mother wavelet is a square integrable function that generates a family of daughter wavelets by scaling and translating operations. Mathematically, it can be expressed as:

$$
\psi_{s, u}(t)=\frac{1}{\sqrt{|s|}} \psi\left(\frac{t-u}{s}\right)
$$

The parameter $s$ is a scaling or dilation factor, controlling the length of the wavelet and $u$ is a location parameter that specifies where the wavelet is centered.

The mother wavelets account for the high frequency or detailed parts on each scale by noting the amount of stretching of the wavelet, while the father wavelet essentially represents the low-frequency component of the time series. The daughter wavelets are generated from the finite length high oscillating mother wavelet through scaling and translation operations. Unlike the mother wavelet, 
the father wavelet is a single elementary waveform which largely determines the quality of transformation. We have used MODWT for decomposition process utilizing the Haar filter. The present study considers six levels of decomposition for executing time-varying inspection. Table 9 explains the time mapping.

Table 9: Time interpretation of different frequencies

\begin{tabular}{|l|l|l|}
\hline Scale 1 & $\mathrm{D}_{1}$ & 2 4 days (Intraweek Scale) \\
\hline Scale 2 & $\mathrm{D}_{2}$ & 4 8 days (Weekly Scale) \\
\hline Scale 3 & $\mathrm{D}_{3}$ & 8 16 days (Fortnightly Scale) \\
\hline Scale 4 & $\mathrm{D}_{4}$ & 16 32 days (Monthly Scale) \\
\hline Scale 5 & $\mathrm{D}_{5}$ & 32 64 days (Monthly to Quarterly Scale) \\
\hline Scale 6 & $\mathrm{D}_{6}$ & 64 128 days (Quarterly to Biannual Scale) \\
\hline
\end{tabular}

Quantile Regression: Generally, to establish a relationship between an independent variable and a set of variables, a model with linear specification is formulated which is conditional upon its mean. Thus, the results essentially focus upon the mean value relationship. However, the relationship at different levels of conditional distribution of the dependent variable remains unexplored. To model the complex dependence of time series, Quantile Regression (QR) technique is applied on time-varying wavelet decomposition. The $\mathrm{QR}$ technique was introduced by Koenker and Bassett (1978), Mensi et al. (2014) and Nusair and Al-Khasawneh (2018) have applied these techniques.

Let $\mathrm{Y}$ be the dependent variable, which is assumed to be dependent on $\mathrm{X}$ linearly. Thus, the $\tau^{t h}$ conditional quantile of a function may be specified as follows:

$$
Q_{Y}(\tau \mid X)=\inf \left(b \mid G_{Y}(b \mid X) \geq \tau\right)=\sum_{k} \beta_{k}(\tau) X_{k}
$$

where the conditional distribution function of $(Y \mid X)$ is represented by $G_{Y}(b \mid X)$; $b$ is an actual realization of $X$.

The relationship of dependence between vector $\mathrm{X}$ and the $\tau^{\text {th }}$ conditional quantile of function is determined by $\beta(\tau)$. The dependence is conditional if the exogenous variables are added to $X$, while the dependence is unconditional if the exogenous variables are excluded from $X$. The complete dependence structure of $\mathrm{Y}$ is determined by $\beta(\tau)$ for $\tau \in[0,1]$. There can be three possible dependence structures between $\mathrm{Y}$ and vector of independent variables $\mathrm{X}$ : (a) where the value of $\beta(\tau)$ for different values of is similar (dissimilar), the relationship is symmetric (asymmetric) for lower and higher quantiles, (b) where the value of $\beta(\tau)$ does not change, the relationship is constant, and (c) where the values of $\beta(\tau)$ increases (decreases) with the values of $\tau$, the relationship is monotonically increasing (decreasing). 
For a given $\tau$, the coefficients of $\beta(\tau)$ are estimated by minimization of the weighted absolute deviations between $\mathrm{Y}$ and $\mathrm{X}$ :

$$
\hat{\beta}(\tau)=\arg \min \sum_{t-1}^{T}\left(\tau-1_{Y_{t}<\sum_{k} \beta_{k}(\tau) X_{k}}\right)\left|Y_{t}-\sum_{k} \beta_{k}(\tau) X_{k}\right|
$$

where $T$ denotes the temporal information whilst the usual indicator function is denoted by $1_{Y_{t}<\sum_{k} \beta_{k}}(\tau) X_{k}$. The solution to this problem is arrived by using a linear programming algorithm (Koenker and D’Orey, 1987). To obtain the standard error for the estimated coefficients, the pair-bootstrapping procedure proposed by Buchinsky (1995) is used. The asymptotically valid standard errors under heteroskedasticity and misspecifications of the $\mathrm{QR}$ function are obtained by this pair-bootstrapping procedure.

Quantile Regression of wavelet-decomposed values of the variables allows to model the interaction dynamics between the variables across different timescales and also at different market conditions. This model has been applied by Das et al. (2018); Karlsson et al. (2017); Mensi et al. (2014).

Wavelet Multiple Correlation and Cross Correlation: To overcome certain shortcomings of traditional pairwise correlation and cross correlation, Fernández-Macho (2012) introduced WMC and WMCC techniques. These use maximal overlap discrete wavelet transformation (MODWT) on a multivariate stochastic process $X_{t}=\left(x_{1 t}, x_{2 t}, \ldots, x_{n t}\right)$ to generate wavelet coefficients $W_{i j t}=\left(w_{1 j t}, w_{2 j t}, \ldots, w_{n j t}\right)$ at respective scales $\left(\lambda_{j}\right)$. The $\mathrm{WMC}\left(\varphi_{x}\left(\lambda_{j}\right)\right)$ represents a set of multi-scale correlation determined from the stochastic process. The square root of regression coefficient of determination associated with the respective scales $\left(\lambda_{j}\right)$ is calculated in linear combination of wavelet coefficients having maximum coefficient of determination. The coefficient of regression of a variable $\left(z_{i}\right)$ on a regressor set $\left(z_{k}, k \neq i\right)$ is estimated as:

$$
R^{2}=1-1 / \rho^{i i}
$$

where $\rho^{i i}$ denotes the $i^{\text {th }}$ diagonal element of the inverse of the complete correlation matrix (Fernández-Macho, 2012). WMC is calculated as:

$$
\varphi_{x}\left(\lambda_{j}\right)=\sqrt{1-\frac{1}{\max \operatorname{diag} P_{j}^{-1}}}
$$

where $P_{j}$ represents the correlation matrix defined on $W_{j t}$ and $\max \operatorname{diag}($. operator chooses the largest element in the diagonal of the argument. The coefficient of determination linked to regression of $z_{i}$ on the remaining variables can be expressed as square of correlation between actual observations $\left(z_{i}\right)$ and estimated values $\left(\hat{z}_{i}\right)$. Hence alternatively, the WMC can also be expressed as: 


$$
\varphi_{x}\left(\lambda_{j}\right)=\frac{\operatorname{Cov}\left(w_{i j t}, \widehat{w}_{i j t}\right)}{\sqrt{\operatorname{Var}\left(w_{i j t}\right) \operatorname{Var}\left(\widehat{w}_{i j t}\right)}}
$$

The wavelet covariance and variance are computed as:

$$
\begin{gathered}
\operatorname{Cov}\left(w_{i j t}, \widehat{w}_{i j t}\right)=\bar{\gamma}_{j}=\frac{1}{T_{j}} \sum_{t=L_{j}^{-1}}^{T-1} w_{i j t} \widehat{w}_{i j t} \\
\operatorname{Var}\left(w_{i j t}\right)=\bar{\delta}_{j}^{2}=\frac{1}{T_{j}} \sum_{t=: j^{-1}}^{T-1} w_{i j t}^{2} \\
\operatorname{Var}\left(\widehat{w}_{i j t}\right)=\bar{\varepsilon}_{j}^{2}=\frac{1}{T_{j}} \sum_{t=: j^{-1}}^{T-1} \widehat{w}_{i j t}^{2}
\end{gathered}
$$

Maximization of the coefficient of determination is driven by $w_{i j}$ on set of regressors $\left\{w_{k j}, k \neq i\right\}$. The fitted values of regression are represented by $\widehat{w}_{i j}$. The number of the affected wavelet coefficients due to the boundary of the wavelet filter having length $\mathrm{L}$ and scale $\lambda_{j}$ is given by:

$$
L_{j}=\left(2^{j}-1\right)(L-1)+1
$$

while the number of unaltered coefficients is governed by the following equation:

$$
\tilde{T}=T-L_{j}+1
$$

The WMCC can be computed by putting a lag $(\tau)$ between the actual and estimated figures of the criterion construct at each scale $\left(\lambda_{j}\right)$. Mathematically, the WMCC is formulated as:

$$
\varphi_{x} \tau\left(\lambda_{j}\right)=\operatorname{Cor}\left(w_{i j t}, \widehat{w}_{i j t+\tau}\right)=\frac{\operatorname{Cor}\left(w_{i j t}, \widehat{w}_{i j t+\tau}\right)}{\sqrt{\operatorname{Var}\left(w_{i j t}\right) \operatorname{Var}\left(\widehat{w}_{i j t+\tau}\right)}}
$$

Confidence intervals can be constructed assuming that $X=\left(X_{1}, \ldots, X_{T}\right)$ represents a realization of equation 16 in terms of a multivariate Gaussian stochastic process. The wavelet coefficients are generated by applying $J^{\text {th }}$ order MODWT to individual time series components.

$$
\widetilde{W}_{j}=\left[\widetilde{W}_{j 0}, \ldots, W_{j, T-1}\right]=\left[\left(\widetilde{w}_{1 j 0}, \ldots, \widetilde{w}_{n j 0}\right), \ldots,\left(\widetilde{w}_{1 j, \frac{T}{2^{j}}-1}\right)\right], j=1, \ldots, J
$$

Finally, the confidence interval of the coefficient of correlation is calculated as:

$$
C I_{1-\alpha}\left(\varphi_{x}\left(\lambda_{j}\right)\right)=\tanh \left[\tilde{z}_{j} \pm \emptyset_{1-\alpha / 2}^{-1} / \sqrt{\frac{T}{2^{j}}-1}\right]
$$

where $\emptyset_{q}^{-1}$ represents $100 \mathrm{q} \%$ point of the standard normal distribution. 
WMC and WMCC techniques have been used to understand the association between the returns of SENSEX and six company stocks during the pre-crisis, crisis and post-crisis periods.

Diebold-Yilmz Spillover (2009): The N-dimensional VAR(p) model is formulated as:

$$
Y_{t}=\phi_{1} Y_{t-1}+\cdots+\phi_{p} Y_{t-p}+\epsilon_{t}
$$

where $\epsilon_{t}$ is i.i.d error component and the coefficients $\left(\phi_{1} \ldots \phi_{p}\right)$ represent the magnitude and direction of interrelationships between the target and the respective independent constructs. The system can also be expressed as a moving average representation:

$$
Y_{t}=\epsilon_{t}+A_{1} \epsilon_{t-1}+A_{2} \epsilon_{t-2}+\cdots
$$

where $A_{i}$ denote the respective coefficients.

The contained information of coefficient matrices linked to spillover can be formulated with H-step ahead forecast error variance decompositions using equation 17:

$$
Y_{t+H}-P\left(Y_{t+H} \mid Y_{t}, Y_{t-1}\right)=\epsilon_{t}+A_{1} \epsilon_{t+H-1}+A_{2} \epsilon_{t+H-2}+\cdots+A_{H-1} \epsilon_{t+1}
$$

where $P\left(Y_{t+H} \mid Y_{t}, Y_{t-1}\right)$ represents the $H$-step ahead forecast at time $t$.

If $\Sigma_{\epsilon}$ represents the covariance matrix of $\epsilon$ and $A_{0}:=I_{N}$, then the covariance matrix of forecast's error can be calculated as:

$$
\Sigma_{\epsilon, H}=\sum_{h=0}^{H-1} A_{h} \Sigma_{\epsilon} A_{h}^{T r}
$$

Diebold and Yilmaz invented the spillover index (SOI) to measure spillovers in terms of contribution of shocks from one variable to another in forecast's error variance using equation 19:

$$
S O I=100 \times \frac{1}{N} \sum_{i=1}^{N} \frac{\sum_{i \neq j} \sum_{h=0}^{H-1}\left(A_{h} L\right)_{i j}^{2}}{\sum_{h=0}^{H-1}\left(A_{h} \Sigma_{\epsilon} A_{h}^{T}\right)_{i i}}=100 \times\left(1-\frac{1}{N} \sum_{i=1}^{N} \frac{\sum_{h}^{H-1}\left(A_{h} L\right)_{i i}^{2}}{\sum_{h=0}^{H-1}\left(A_{h} \Sigma_{\epsilon} A_{h}^{T}\right)_{i i}}\right)
$$

where $N$ denotes the number of variables under consideration, $L$ is the lower-triangular Cholesky factor of $\Sigma_{\epsilon}$.

We have used this spillover analysis technique to measure the extent of spillovers from SENSEX returns to returns from other chosen assets across the time horizons.

Performing granular Diebold-Yilmaz spillover analysis in conjunction with wavelet decomposition assists in effective critical evaluation of the dynamics of spillover transmission in terms of magnitude and duration. Using the said framework effort has been made to capture short, medium and long run volatility contagion patterns during the pre-crisis, crisis and post-crisis periods. 


\section{Results and analysis}

\subsection{Dependence analysis}

Quantile regression $(\mathrm{QR})$ has been performed on the decomposed time series to understand the dynamic dependence of MID CAP and SMALL CAP sectors on market sentiment on four quantiles $(0.05,0.1,0.9,0.95)$. The three lower quantiles, i.e. 0.05 and 0.1 , represent the bearish market state while the upper quantiles, 0.9 and 0.95 , denote the bullish phase.

In order to test the robustness of our approach, our data set spans four periods, namely: a bullish phase prior to the 2008 world financial crisis, a bearish phase during the crisis, a bullish phase during 2015 and a bearish phase in 2016. While the wavelet method decomposed the aggregate time series data into period-wise movements, ranging from one week to six months, the quantile regression method identified the upswings and downswings within each of the four periods. The following two-way tables for each of the periods summarize the results.

Table 10: Dependence structure during Pre-Crisis

\begin{tabular}{|c|c|c|c|c|}
\hline & \multicolumn{2}{|c|}{ Downswing } & \multicolumn{2}{|c|}{ Upswing } \\
\hline SMALL CAP & .05 & .1 & .9 & .95 \\
\hline D1 & & & & \\
\hline D3 & .58 & & & \\
\hline D6 & .79 & .75 & .31 & .19 \\
\hline
\end{tabular}

We can observe from Table 10 that the effects of SENSEX returns on MID CAP returns are much stronger during downswings than in upswings in the very short run, i.e. two to four days, and also within a fortnight. This effect gets stronger as time progresses beyond three months. This is also true of the effect of SENSEX returns on SMALL CAP returns. However, during the upswing, the effect wanes after a fortnight. The effect on SMALL CAP returns falls drastically. The results indicate that in a downswing, that is with bad news, SMALL CAP and MID CAP stock returns take a beating immediately. During an upswing, however, they do not move with market sentiment after a couple of months. So there is an asymmetry in effects of bad news and good news on such stock returns. 
Table 11: Dependence structure during Crisis

\begin{tabular}{|c|c|c|c|c|}
\hline & \multicolumn{2}{|c|}{ Downswing } & \multicolumn{2}{|c|}{ Upswing } \\
\hline SMALL CAP & .05 & .1 & .9 & .95 \\
\hline D1 & .57 & .55 & .57 & \\
\hline D3 & & & & \\
\hline D6 & 1.31 & 1.22 & 1.26 & 1.25 \\
\hline
\end{tabular}

Table 11 summarizes the extent and duration of the effect of market sentiment on the MID CAP and SMALL CAP stocks during the 2008 financial crisis. It can be observed that first, the long term effects were much more severe than those in the pre-crisis phase. Second, the long term effects were stronger than the short term effects within this period. Third, these stocks did not respond to any market corrections within the crisis period. Fourth, there is no significant difference in the effects of market shocks on these two classes of stocks.

Table 12: Dependence structure during the Post-Crisis Bearish phase

\begin{tabular}{|c|c|c|c|c|}
\hline & \multicolumn{2}{|c|}{ Downswing } & \multicolumn{2}{|c|}{ Upswing } \\
\hline$\underbrace{\text { MID CAP }}_{\text {SMALL CAP }}$ & .05 & .1 & .9 & .95 \\
\hline D1 & $\mathrm{T}_{1.01} \longrightarrow 1.03$ & ${ }_{1.04} \longrightarrow .98$ & $\mathrm{C}_{1}^{1}$ & ${ }_{1.02}{ }^{1.1}$ \\
\hline D3 & ${ }_{1.12}-1.07$ & ${ }_{1.15}-1.14$ & $\mathrm{C}_{1}{ }^{1.02}$ & ${ }_{98}{ }^{1}{ }^{1}$ \\
\hline D6 & ${ }_{1.54}-1.28$ & ${ }_{1.29} \longrightarrow 1.13$ & $\begin{array}{l}1.49 \\
\longrightarrow\end{array}$ & ${ }_{1.60} \longrightarrow 1.26$ \\
\hline
\end{tabular}

In this post-crisis bearish phase during 2015, the effect of market sentiment has been quite severe in both the MID CAP and SMALL CAP segments, over a significant time period, as reported in Table 12. The magnitudes are quite high, and in the short run, the effect on the SMALL CAP segment has been relatively more severe than on the MID CAP stocks. In the upswing phase, in this overall bearish market sentiment, both sets of stocks responded positively, and the long term effects were stronger sensing a market reversal. 
Table 13: Dependence structure during the Post-Crisis Bullish phase

\begin{tabular}{|c|c|c|c|c|}
\hline & \multicolumn{2}{|c|}{ Downswing } & \multicolumn{2}{|c|}{ Upswing } \\
\hline${ }_{\text {SMALL CAP }}^{\text {MID CAP }}$ & .05 & .1 & .9 & .95 \\
\hline D1 & 1.12 & & $\mathbf{Y}^{.77}$ & 1.04 \\
\hline D3 & & & .89 & .93 \\
\hline D6 & 1.01 & 1.07 & $\begin{array}{l}.98 \\
.78\end{array}$ & .74 \\
\hline
\end{tabular}

Table 13 suggests that even in a bullish phase, during downward correction, the SMALL CAP stocks get affected more than the MID CAP stocks. Furthermore, between upswing and downswing, there is an asymmetry in effects for the SMALL CAP stocks. The returns from such stocks are not as sensitive to positive news as they are to negative news. The results indicate that for the MID CAP stocks, the effects of bad news or good news were similar across time spans.

Thus the overall findings of QR assist us in comprehending the time-varying nature of the influence of market sentiments on sectoral level stock returns during pre-crisis, crisis, and post-crisis periods.

\subsection{WMC \& WMCC}

As explained earlier, WMC and WMCC have been applied on SENSEX returns of (SN), BF, CP, GD, BC, TC and GM to study the overall dynamic interaction in phase-wise manner. Basically, they extract the nature co-movement and the leader responsible for driving the association. The following figures present the outcome of WMC and WMCC analysis, respectively.

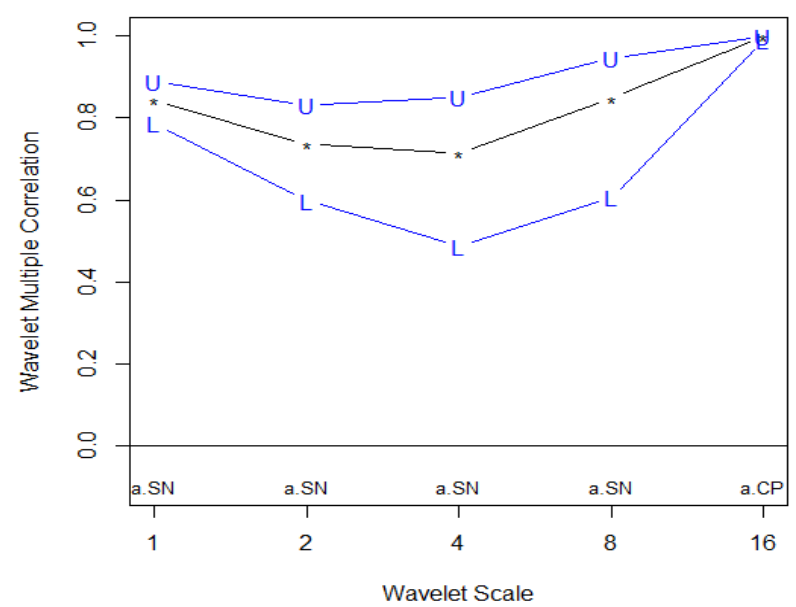

Figure 1: WMC plot of the Pre-Crisis phase 
The wavelet scale 1 to 5 accounts for D1 to D5. The WMC plot reveals that at D1 and D5 scales, the correlation coefficient attains its local and global maxima values, respectively. From D1 to D3, the correlation decreases, while from D3 to D5 it increases monotonically. Hence it can be concluded that during the pre-crisis period, a high degree of association existed among the considered assets initially in the short run. The interaction gradually decayed slowly from short run to medium run; it gained momentum again from medium to long term. It can be seen that $\mathrm{SN}$ was leading on the D1 to D4 scale, while $\mathrm{CP}$, on the D5 scale.

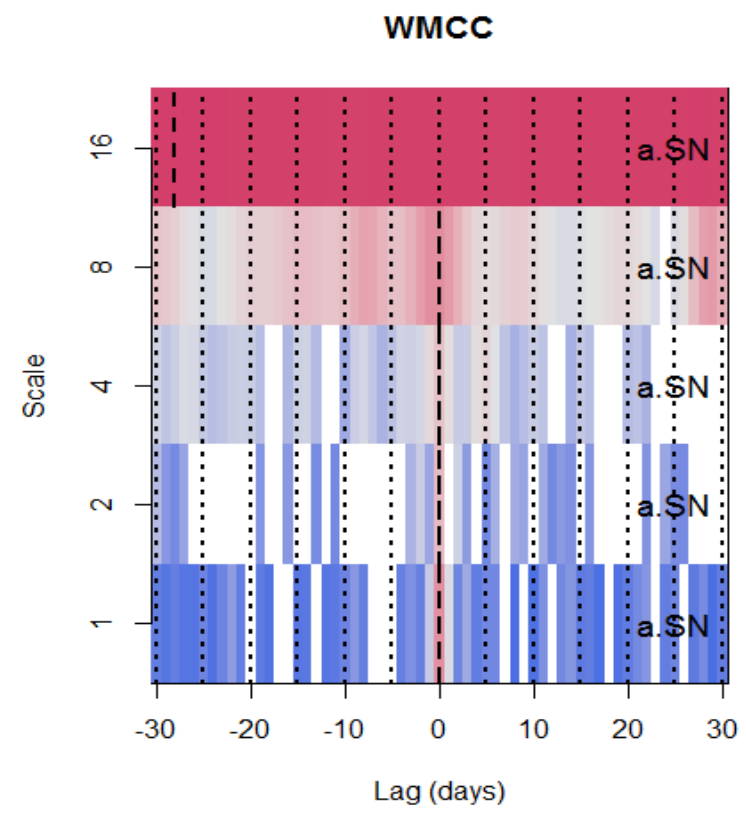

Figure 2: WMCC plot of the Pre-Crisis phase

The WMCC plots further explore the association at various lag lengths apart from point-wise estimation. The plots are generated using Daubechies 'la8' filter allowing a time lag of one month (30 days). The localization of the highest wavelet correlation is indicated by vertical dashed lines. Different colors are used to measure the strength of association: shaded region represents strongest association, whereas blank region implies no correlation.

The WMCC figure of the pre-crisis period suggests the existence of strong interaction localization at the largest scale, D5, which persisted for a lag of up to one month approximately. SENSEX emerged as the market leader across all the scales. 


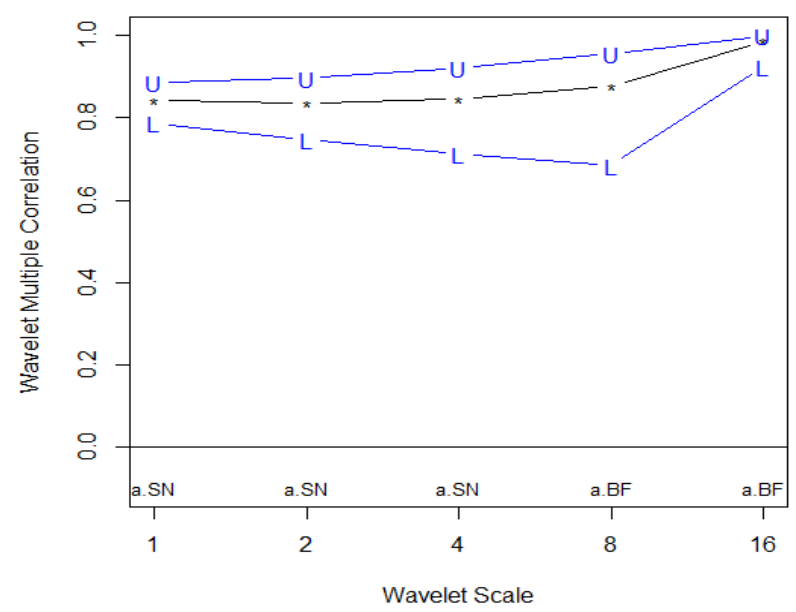

Figure 3: WMC plot of the Crisis phase

The WMC plot indicates a high degree of interaction as manifested through the correlation coefficient prevailing throughout the crisis period. At the D5 scale, i.e., in long run, it became maximum. Hence, it can be inferred that during this period, the interaction got stronger with time. SN emerged as the dominant driver of the association at the D1, D2 and D3 scales, while BF was the leader at the D4 and D5 scales. The distance between the upper and lower bands narrowed and the actual association also did not fall with time.

\section{WMCC}

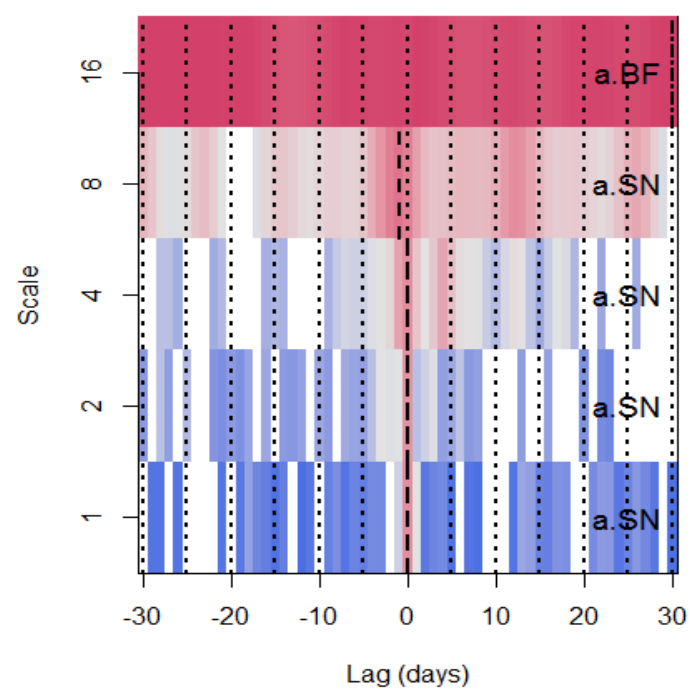

Figure 4: WMCC plot of the Crisis phase 
The WMCC plot of the crisis period points out the presence of a highest degree of association mostly in the longest scale, i.e., D5, for a lag of up to nearly one month. SN emerged as the leader in the D1 to D4 scales, while BF acted as leader in the D5 scale.

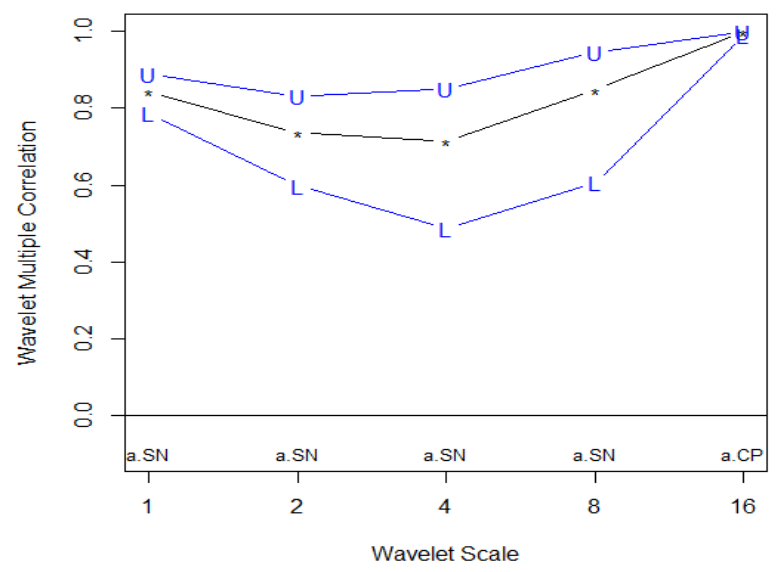

Figure 5: WMC plot of the Post-Crisis downswing phase

During the post-crisis downswing period, the WMC plot reveals that the association nexus initially marginally decreased from D1 to D3 scale. Then from D3 to D5 scale, the interaction again gradually gained momentum as a steep increase in the correlation coefficient is apparent. Hence during the post-crisis downswing phase, the seven assets quickly entered into high interaction phase which gradually faded to fortnightly scale. Again, from the fortnightly to the quarterly period the interaction gradually became intense. SN and $\mathrm{CP}$ emerged as the leaders at D1-D4 and D5 scales, respectively.

WMCC

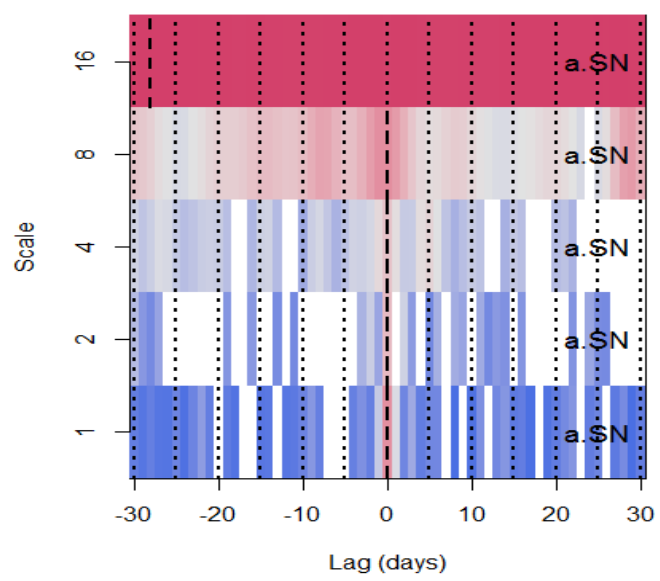

Figure 6: WMCC plot of the Post-Crisis downswing phase 
Similarly to the pre-crisis and post-crisis phases, during the downswing phase of the post-crisis period, the WMCC plot reveals strong presence of a correlation among the seven financial time series across a lag period of one month in the D5 scale. SN has been found to be the leader across all time scales.

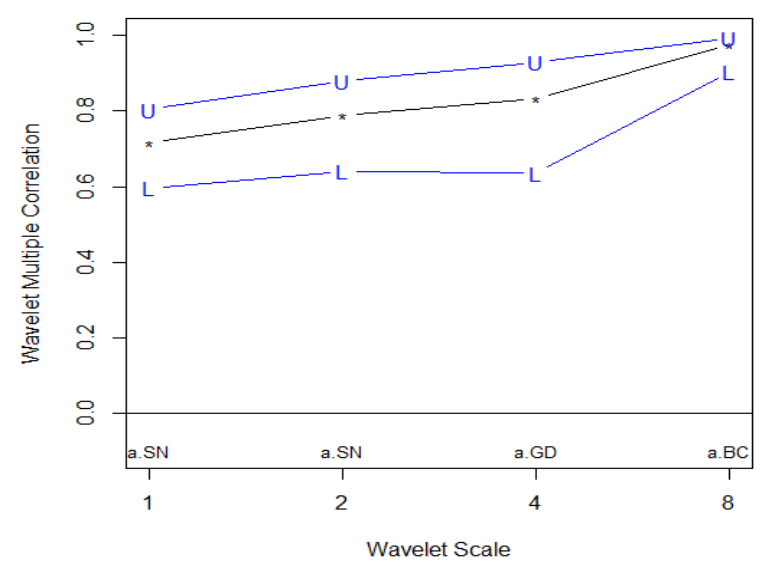

Figure 7: WMC plot of the Post-Crisis upswing phase

The plot suggests that during the post crisis upswing period, the association among the seven financial time series gradually became stronger from the D1 to D4 scale, i.e., from short to medium run duration. Two new entrants, i.e. GD and $\mathrm{BC}$, are found to lead the association at the D3 and D4 scales, while SN remained the leader at the D1 and D2 scales.

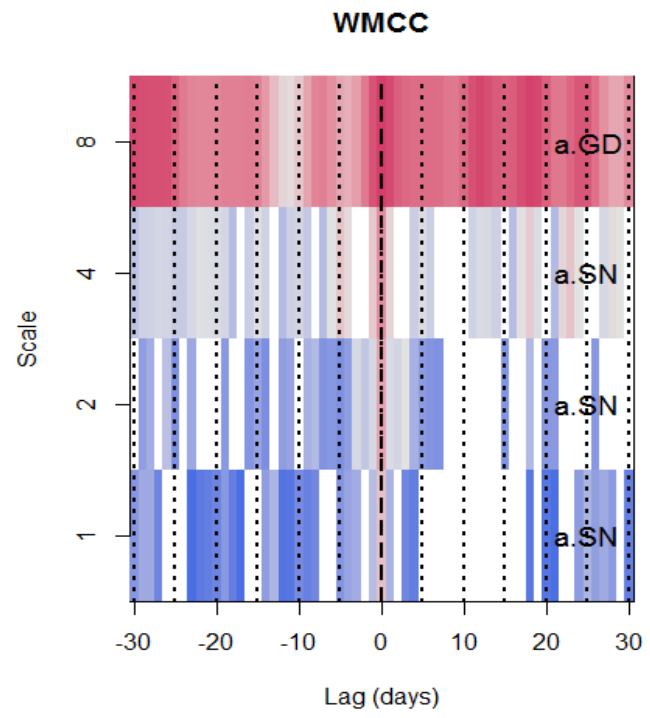

Figure 8: WMCC plot of the Post-Crisis upswing phase 
During the upswing period of the post-crisis phase, the WMCC figure suggests the strongest concentration of association in the D4 scale. However, the cross correlation is unable to find the exact duration of the lag periods, as the spread of the association is not uniform and appears to be fading. GD has been found to drive cross correlation at the D4 scale, while SN leads them in the remaining scales.

Hence, it can be concluded that market sentiment had a profound association with six company stocks during different time phases. Market sentiment has also been found to be leading the association at distinct time scales. The insights generated through WMC and WMCC can be effectively exploited for time-varying portfolio diversification. In a nutshell, the following inferences can be drawn:

- In the upswing phase of the pre sub-prime crisis, markets were on the high and rising. There was herding and the association increased with time. However, in the short run there was portfolio realignment and these stocks may not have found favor. The dispersion between the upper and lower bounds becomes high, indicating portfolio alignment. But in the long run, every stock was good, implying that in the presence of herding, market participants paid scant attention to the fundamentals of stocks and bought indiscriminately, feeling that every stock would give significant positive returns.

- During the crisis phase, there was severe herding from the very beginning, and as the crisis deepened, the association increased. The dispersion was also much lower, showing that the market was sliding uniformly and fast. There was no time for portfolio alignment.

- In the downswing, there was portfolio realignment, and these stocks do not show association. However, as time progressed, some herding can be seen in the long run. Markets fell, and so did returns from these stocks. Portfolio realignment led to an increase in the dispersion, which decreased with time, as herding set in.

- In the next upswing phase, a bullish phase, the market was looking good. So all the stocks moved up together and as the upswing persisted, the association also improved. This is also evident from the fact that the dispersion was much lower than in the downswing phase.

\subsection{Diebold-Yilmaz spillover analysis}

One of the objectives of the paper is to understand volatility spillover of market sentiment on sectoral returns and company stock returns. We have applied Diebold-Yilmaz spillover analysis on decomposed components of the respective series of returns to measure the volatility spillovers. We report the level of 
spillovers at three levels, D2, D4, and D6, which act as proxies for short, medium and long run durations. The following tables summarize spillover percentage during the pre-crisis, crisis, and post-crisis phases.

Table 14: Spillover during the PRE-CRISIS phase

\begin{tabular}{|c|r|r|r|r|r|r|r|r|}
\hline Scale & \multicolumn{1}{|c|}{ SMALL CAP } & \multicolumn{1}{c|}{ MID CAP } & \multicolumn{1}{c|}{ BF } & \multicolumn{1}{c|}{ CP } & \multicolumn{1}{c|}{ GD } & \multicolumn{1}{c|}{ BC } & \multicolumn{1}{c|}{ TC } & GM \\
\hline D2 & 31.59 & 37.88 & 11.23 & 3.85 & 51.86 & 15.74 & 1.16 & 10.14 \\
\hline D4 & 31.56 & 37.20 & 2.89 & 29.16 & 27.22 & 22.60 & 10.35 & 16.76 \\
\hline D6 & 46.30 & 28.73 & 6.67 & 14.81 & 7.84 & 2.27 & 0.89 & 11.22 \\
\hline
\end{tabular}

It can be seen that during the pre-crisis phase, a significant amount of volatility was transmitted from SENSEX to both SMALL CAP and MID CAP in the short, medium and long runs. During the long run, the maximum amount of volatility transmission could be observed from the market to the SMALL CAP sector, while MID CAP received maximum spillover in the short run. In the short and medium time spans, volatility contagion between SENSEX and MIDCAP was more intense than between SENSEX and SMALL CAP. Among the company stocks, GD received the maximum impact from SENSEX in the short run, whereas $\mathrm{CP}$ was the maximum recipient in the medium run. In the long run, the amount of spillovers from market sentiment to all company stocks dwindled. This was a bullish phase, and for established companies, fundamentals became important rather than short-term volatility.

Table 15: Spillover during the CRISIS phase

\begin{tabular}{|c|r|r|r|r|r|r|r|r|}
\hline Scale & \multicolumn{1}{|c|}{ SMALL CAP } & \multicolumn{1}{c|}{ MID CAP } & \multicolumn{1}{c|}{ BF } & \multicolumn{1}{c|}{ CP } & GD & \multicolumn{1}{c|}{ BC } & \multicolumn{1}{c|}{ TC } & \multicolumn{1}{c|}{ GM } \\
\hline D2 & 27.26 & 43.05 & 3.88 & 1.86 & 34.45 & 7.11 & 2.45 & 9.41 \\
\hline D4 & 60.65 & 62.16 & 0.84 & 2.85 & 41.69 & 16.89 & 5.71 & 2.28 \\
\hline D6 & 30.93 & 35.92 & 0.26 & 29.36 & 20.28 & 5.43 & 18.23 & 6.37 \\
\hline
\end{tabular}

In the crisis phase, large spillovers can be observed and both the MID CAP and SMALL CAP indices received maximum spillover in the medium run. SMALL CAP received comparatively minimum spillover in the short run. That is, once the global financial crisis hit the markets, the MID CAP sector suffered the immediate brunt; then it intensified and spilled over to the SMALL CAP sector. It can be concluded that contagion was stronger in the medium run between market sentiments and sectoral indices. Considering the individual companies, only GD was affected, and to some extent CP. 
Table 16: Spillover during the POST-CRISIS downswing phase (2015)

\begin{tabular}{|c|r|r|r|r|r|r|r|r|}
\hline Scale & \multicolumn{1}{|c|}{ SMALL CAP } & \multicolumn{1}{c|}{ MID CAP } & \multicolumn{1}{c|}{ BF } & \multicolumn{1}{c|}{ CP } & \multicolumn{1}{c|}{ GD } & \multicolumn{1}{c|}{ BC } & \multicolumn{1}{c|}{ TC } & \multicolumn{1}{c|}{ GM } \\
\hline D2 & 41.75 & 22.35 & 1.54 & 0.44 & 49.93 & 29.08 & 0.02 & 15.72 \\
\hline D4 & 13.43 & 25.92 & 3.25 & 12.15 & 24.84 & 51.27 & 1.08 & 2.91 \\
\hline D6 & 49.24 & 61.47 & 12.70 & 72.41 & 1.14 & 0.10 & 10.51 & 0.29 \\
\hline
\end{tabular}

In the post-crisis downswing phase the SMALL CAP sector received the highest spillover in the short run and also in the long run. The effect intensified for the MID CAP sector in the long run. In the case of individual companies, $\mathrm{GD}$ and $\mathrm{BC}$ were affected by the market in the short and medium run, and $\mathrm{CP}$ was most affected in the long run.

Table 17: Spillover during the POST-CRISIS upswing phase (2016)

\begin{tabular}{|c|r|r|r|r|r|r|r|r|}
\hline Scale & \multicolumn{1}{|c|}{ SMALL CAP } & \multicolumn{1}{c|}{ MID CAP } & \multicolumn{1}{c|}{ BF } & \multicolumn{1}{c|}{ CP } & \multicolumn{1}{c|}{ GD } & \multicolumn{1}{c|}{ BC } & TC & \multicolumn{1}{c|}{ GM } \\
\hline D2 & 26.33 & 26.39 & 4.85 & 4.32 & 78.84 & 3.06 & 1.13 & 7.18 \\
\hline D4 & 35.97 & 58.04 & 0.35 & 38.26 & 1.70 & 4.54 & 8.21 & 1.25 \\
\hline D6 & 54.04 & 49.18 & 21.97 & 0.34 & 19.73 & 6.40 & 2.05 & 17.80 \\
\hline
\end{tabular}

During the post-crisis upswing phase, spillovers received by SMALL CAP and MID CAP gradually increased from the short run to the long run. Among company stocks, GD and $\mathrm{CP}$ once again emerged as the top recipients of spillovers in the short run and medium run, respectively, while BF received maximum spillover in the long run.

It can be concluded that we have successfully captured and interpreted the interplay of financial markets represented by SMALL CAP, MID CAP, market sentiment and six company stocks through proper deployment of research methodologies. The present paper constitutes a significant contribution to existing research. The overall findings indicate that the relationships do not follow a uniform pattern across different timescales (Das et al., 2018; Ghosh and Datta Chaudhuri, 2019).

\section{Conclusion}

The performance of different sectors of an economy reflects the demand pattern of the consumers, both national and global. This manifests itself in the performance of the companies in the different sectors, and consequently gets reflected in their share prices. Furthermore, there are companies of different sizes which can be classified by their market capitalization, namely large cap, mid cap and small cap companies. Following the principles of microeconomics, the size of a company depends on the prevailing competitive position and 
economies of scale. Our focus in this paper is to examine to what extent overall market-wide shocks affect the different sectors and also companies of various sizes. The study also considers these effects over different time periods to understand whether the intensity and duration of the effect of shocks undergo any change.

The analysis is important as companies, whose share prices get affected by shocks, may find it hard in the short run to raise resources from the market, which in turn can adversely affect their growth prospects. Thus, the study attempts to measure the extent of spillover of the shocks and also the duration of the shocks across sectors and companies over different time periods.

In this paper, we study the effect of overall stock market sentiment in India on sectoral indices and on individual stock prices in terms of co-movement, dependence and volatility transmission along with the magnitude and persistence of the effects. The study uses a wavelet decomposition framework for breaking down different financial time series into time-varying components. Quantile Regression, Wavelet Multiple Correlation and Cross-Correlation analysis, and Diebold-Yilmaz spillover analysis are then applied to investigate the nature of dependence, association, and spillover dynamics. For further focus, we have considered different time periods separately to identify the effect of market phases. Interesting results are obtained with respect to persistence of shocks, both across time periods and within time periods. These have implications for the understanding of market behavior and also perception of sectors and stocks.

The contribution of the present study can be segregated into three broad strands. First, it analyzes the dynamic interplay among the variables at a granular level to understand the effects in the short, medium, and long run. The interplay has been decoded through careful investigation of dependence structure, co-movement dynamics, and volatility contagion effects. The methodological frameworks include quantile regression, wavelet driven correlation, and Diebold-Yilmaz spillover analysis in conjunction with wavelet decomposition to capture dependence, co-movement, and spillover relationship, respectively. Second, the paper analyzes four different time periods. Unlike the majority of previous studies dedicated to examining interrelationships in the pre-crisis, crisis, and post-crisis phases at aggregate level, the present paper further splits the post-crisis phase into downswing and upswing phases for drawing inferences. The regime-driven exploration in association with wavelet-based time-frequency modeling delivers key insights and rationalizes the positioning of the paper with respect to past literature. Third, the paper looks at companies of different sizes and also sectoral indices in terms of market capitalization. 
The paper brings out the time-varying nature of association, dependence and volatility spillover dynamics of Indian stock market using wavelet-based frameworks. The results reveal the nature of interaction during the global financial crisis, pre-crisis and post-crisis phases. It has been observed that market sentiment has played a pivotal role in influencing both sectoral level stock indices and company level stock returns during the specified time periods. However, the magnitude of the impact has not been homogenous. The key insights generated from our granular level analysis are as follows:

- In terms of dependence, during the pre-crisis period, a comparatively stronger dependence of the MID CAP and SMALL CAP sectors on market sentiment has been detected in the bearish phase than in the bullish phase.

- During the crisis phase, the long run dependence was stronger than the short and medium run in both bearish and bullish market states.

- In the post-crisis downswing period, the dependence structure between market sentiment and SMALL CAP has been noticed to be relatively stronger than market sentiment and the MID CAP counterpart. A similar scenario prevailed during the post crisis upswing phase as well.

- In the context of association between market sentiment and six company stocks, our framework reveals that during the pre-crisis period there existed considerable opportunities for portfolio diversification.

- In the sub-prime crisis phase the association became extremely strong between market sentiment and stocks of the chosen companies, which implied very little opportunity for portfolio diversification.

- During the post-crisis downswing phase, evidence of herding behavior has been discovered in the long run time scale. The association bond became stronger during the subsequent upswing phase.

- As per the wavelet based spillover analysis, strong volatility spillover from market sentiment to the MID CAP sector was noticed in the short and medium duration during the pre-crisis bullish period. SMALL CAP was marginally less sensitive to shocks as compared to MID CAP in the short and medium run. Among the companies, GD and CP were the top recipients of volatility shocks. A similar phenomenon was noticed in the crisis phase.

- During both post-crisis downswing and upswing phases SMALL CAP and MID CAP received significant volatility spillover from market sentiment. Apart from $\mathrm{CP}$ and $\mathrm{GD}, \mathrm{BF}$ and $\mathrm{BC}$ received a high degree of volatility.

The granular frameworks have successfully decoded the interaction dynamics of the Indian stock market in phase-wise time-varying manner in the bullish and bearish market states explicitly. The insights can be effectively exploited for practical applications including portfolio design and alignment and policy 
formations in a dynamic manner. The scope of the present study is restricted to six chosen companies. The framework can easily be extended to other Indian companies and other sectoral indices for modeling dynamic dependence structure. In the future, the impact of the COVID-19 pandemic on the Indian stock market in the form of association, dependence and spillover will be investigated.

\section{References}

Bessler W., Beyenbach J., Rapp M.S., Vendrasco M. (2021), The Global Financial Crisis and Stock Market Migrations: An Analysis of Family and Non-family Firms in Germany, International Review of Financial Analysis, 74, 101692, 1057-5219.

Buchinsky M. (1995), Estimating the Asymptotic Covariance Matrix for Quantile Regression Models a Monte Carlo Study, Journal of Econometrics, 68, 303-338.

Chang C.L., McAleer M., Wang Y.A. (2020), Herding Behaviour in Energy Stock Markets during the Global Financial Crisis, SARS, and Ongoing COVID-19*, Renewable and Sustainable Energy Reviews, 134, 110349, p. 1-15.

Choi S.Y. (2021), Analysis of Stock Market Efficiency during Crisis Periods in the US Stock Market: Differences between the Global Financial Crisis and COVID-19 Pandemic, Physica A: Statistical Mechanics and Its Applications, 574, 125988, p. 1-20.

Dai X., Wang Q., Zha D., Zhou D. (2020), Multi-scale Dependence Structure and Risk Contagion between Oil, Gold, and US Exchange Rate: A Wavelet-based Vine-copula Approach, Energy Economics, 88, 104774, p. 1-58.

Das D., Kannadhasan M. (2018), Do Global Factors Impact Bitcoin Prices? Evidence from Wavelet Approach, Journal of Economic Research, 23, 227-264.

Das D., Kannadhasan M., Al-Yahyee K.H., Yoon S.M. (2018), A Wavelet Analysis of Co-Movements in Asian Gold Markets, Physica A: Statistical Mechanics and Its Applications, 492, 192-206.

Datta Chaudhuri T., Ghosh I. (2015), Using Clustering Method to Understand Indian Stock Market Volatility, arXiv:1604.05015.

Datta Chaudhuri T., Ghosh I. (2016), Artificial Neural Network and Time Series Modeling Based Approach to Forecasting the Exchange Rate in a Multivariate Framework, arXiv:1607.02093.

Diebold F.X., Yilmaz K. (2009), Measuring Financial Asset Return and Volatility Spillovers, with Application to Global Equity Markets, The Economic Journal, 119, 158-171.

Fernández-Macho J. (2012), Wavelet Multiple Correlation and Cross-correlation: A Multiscale Analysis of Eurozone Stock Markets, Physica A: Statistical Mechanics and Its Applications, 391, 1097-1104.

Gao H.L., Mei D.C. (2019), The Correlation Structure in the International Stock Markets during Global Financial Crisis, Physica A: Statistical Mechanics and Its Applications, 534, 122056, p. 1-8.

Ghosh I., Datta Chaudhuri T. (2016), Understanding and Forecasting Stock Market Volatility through Wavelet Decomposition, Statistical Learning and Econometric Methods, SSRN Electronic Journal, DOI: 10.2139/ssrn.2930876.

Ghosh I., Datta Chaudhuri T. (2017), Fractal Investigation and Maximal Overlap Discrete Wavelet Transformation (MODWT)-based Machine Learning Framework for Forecasting Exchange Rates, Studies in Microeconomics, 5, 105-131. 
Ghosh I., Datta Chaudhuri T. (2018), Stock Market Portfolio Construction: A Four-stage Model Based on Fractal Analysis, South Asian Journal of Management, 25, 117-149.

Ghosh I., Datta Chaudhuri T. (2019), A Wavelet Approach towards Examining Dynamic Association, Causality and Spillovers, International Journal of Data and Network Science, 3, 23-36.

Ghosh I., Jana R.K., Sanyal M.K. (2019), Analysis of Temporal Pattern, Causal Interaction and Predictive Modeling of Financial Markets Using Nonlinear Dynamics, Econometric Models and Machine Learning Algorithms, Applied Soft Computing, 82, 105553, p. 1-17.

Ghosh I., Sanyal M.K., Jana R.K. (2018), Fractal Inspection and Machine Learning-based Predictive Modelling Framework for Financial Markets, Arabian Journal for Science and Engineering, 43, 4273-4287.

Ghosh I., Sanyal M.K., Jana R.K. (2021), Co-movement and Dynamic Correlation of Financial and Energy Markets: An Integrated Framework of Nonlinear Dynamics, Wavelet Analysis and DCC-GARCH, Computational Economics, 57, 503-527.

Glensk B., Ganczarek-Gamrot A., Trzpiot G. (2013), Portfolio Analysis on Polish Power Exchange and European Energy Exchange, Multiple Criteria Decision Making, 8, 18-30.

Goodell J.W., Goutte S. (2020), Co-movement of COVID-19 and Bitcoin: Evidence from Wavelet Coherence Analysis, Finance Research Letters, https://doi.org/10.1016/j.frl.2020.101625.

Jammazi R., Ferrer R., Jareno F., Shahzad J. (2017), Time-varying Causality between Crude Oil and Stock Markets: What Can We Learn from a Multiscale Perspective? International Review of Economics \& Finance, 49, 453-483.

Jiang Z., Yoon S.M. (2020), Dynamic Co-movement between Oil and Stock Markets in Oil-importing and Oil-exporting Countries: Two Types of Wavelet Analysis, Energy Economics, 90, 104835, p. 1-33.

Juszczuk P., Kaliszewski I., Miroforidis J. (2017), Trade-Off Guided Search for Approximate Pareto Optimal Portfolios, Multiple Criteria Decision Making, 12, 49-59.

Karlsson H.K., Karlsson P., Månsson K., Sjölander P. (2017), Wavelet Quantile Analysis of Asymmetric Pricing on the Swedish Power Market, Empirica, 44, 249-260.

Koenker R., Bassett G. (1978), Regression Quantiles, Econometrica Journal of the Econometric Society, 46, 33-50.

Koenker R.W., D’Orey V. (1987), Algorithm AS 229: Computing Regression Quantiles, Journal of the Royal Statistical Society. Series C (Applied Statistics), 36, 383-393.

Liu X., An H., Huang S., Wen S. (2017), The Evolution of Spillover Effects between Oil and Stock Markets across Multi-scales Using Wavelet Based GARCH-BEKK model, Physica A: Statistical Mechanics and Its Applications, 465, 374-383.

Mensi W., Hammoudeh S., Reboredo J.C., Nguyen D.K. (2014), Do Global Factors Impact BRICS Stock Markets? A Quantile Regression Approach, Emerging Markets Review, 19, 1-17.

Mishra S., Sharif A., Khuntia S., Meo M.S., Abdul S., Khan R. (2019), Does Oil Prices Impede Islamic Stock Indices? Fresh Insights from Wavelet-based Quantile-on-Quantile Approach, Resources Policy, 62, 292-304.

Mo B., Chen C., Nie H., Jiang Y. (2019), Visiting Effects of Crude Oil Price on Economic Growth in BRICS Countries: Fresh Evidence from Wavelet-based Quantile-on-quantile Tests, Energy, 178, 234-251.

Nikkinen J., Piljak V., Rothovius T. (2020), Impact of the 2008-2009 Financial Crisis on the External and Internal Linkages of European Frontier Stock Markets, Global Finance Journal, 100481, p. 1-9.

Nusair S.A., Al-Khasawneh J.A. (2018), Oil Price Shocks and Stock Market Returns of the GCC Countries: Empirical Evidence from Quantile Regression Analysis, Economic Change and Restructuring, 51, 339-372. 
Polanco-Martinez J.M., Fernandez-Macho J., Neumann M.B., Faria S.H. (2018), A Pre-crisis vs. Crisis Analysis of Peripheral EU Stock Markets by Means of Wavelet Transform and a Nonlinear Causality Test, Physica A: Statistical Mechanics and Its Applications, 490, 1211-1227.

Sharif A., Aloui C., Yarovaya L. (2020), COVID-19 Pandemic, Oil Prices, Stock Market, Geopolitical Risk and Policy Uncertainty Nexus in the US Economy: Fresh Evidence from the Wavelet Based Approach, International Review of Financial Analysis, 70, 101496, p. 1-9.

Yamani E. (2021), Foreign Exchange Market Efficiency and the Global Financial Crisis: Fundamental versus Technical Information, The Quarterly Review of Economics and Finance, 79, 74-89. 